\title{
Epigenetically-Regulated MicroRNA-9- 5p Suppresses the Activation of Hepatic Stellate Cells via TGFBR1 and TGFBR2
}

\author{
Fujun Yu ${ }^{a}$ BiCheng Chen ${ }^{b} \quad$ XuFei Fan ${ }^{c}$ Guojun Lid Peihong Dong ${ }^{\mathrm{e}}$ \\ Jianjian Zheng ${ }^{\mathrm{b}}$
}

\begin{abstract}
aDepartment of Gastroenterology, The First Affiliated Hospital of Wenzhou Medical University, Wenzhou, 'bey Laboratory of Hepatobiliary and Pancreatic Surgery, The First Affiliated Hospital of Wenzhou Medical University, Wenzhou, 'Emergency Department, The First Affiliated Hospital of Wenzhou Medical University, Wenzhou, dDepartment of Hepatology, Ningbo Yinzhou Second Hospital, Ningbo; 'Department of Infectious Diseases, The First Affiliated Hospital of Wenzhou Medical University, Wenzhou, China
\end{abstract}

\section{Key Words}

MicroRNA-9-5p • DNA methylation • Hepatic stellate cell • TGFBR1 • TGFBR2

\begin{abstract}
Background/Aims: Recently, microRNAs (miRNAs) have been demonstrated to act as regulators of activation of hepatic stellate cells (HSCs). It is well known that the main profibrogenic inducer transforming growth factor- $\beta 1$ (TGF- $\beta 1$ ) contributes to HSC activation, which is a key event in liver fibrosis. Increasing studies show that miR-9-5p is down-regulated in liver fibrosis and restoration of miR-9-5p limits HSC activation. However, the role of miR9-5 $p$ in TGF- $\beta 1$-induced HSC activation is still not clear. Methods: miR-9-5p expression was quantified using real-time PCR in chronic hepatitis $B(C H B)$ patients and TGF- $\beta 1$-treated LX-2 cells. In CHB patients, histological activity index (HAI) and fibrosis stages were assessed using the Ishak scoring system. Effects of miR-9-5p on liver fibrosis in vivo and in vitro were analyzed. Luciferase activity assays were performed to examine the binding of miR-9-5p to the 3'-untranslated region of type I TGF- $\beta$ receptor (TGFBR1) as well as TGFBR2. Results: Compared with healthy controls, miR-9-5p was reduced in CHB patients. There was a lower miR-9-5 $p$ expression in CHB patients with higher fibrosis scores or HAI scores. miR-9-5p was down-regulated by TGF- $\beta 1$ in a dose-dependent manner. TGF- $\beta 1$-induced HSC activation including cell proliferation, $\alpha$-SMA and collagen expression was blocked down by miR-9$5 p$. Notably, miR-9-5p ameliorates carbon tetrachloride-induced liver fibrosis. As determined by luciferase activity assays, TGFBR1 and TGFBR2 were targets of miR-9-5p. Further studies demonstrated that miR-9-5p inhibited TGF- $\beta 1 /$ Smads pathway via TGFBR1 and TGFBR2. Interestingly, promoter methylation was responsible for miR-9-5p down-regulation in liver fibrosis. The relationship between miR-9-5p expression and methylation was confirmed in CHB patients and TGF- $\beta 1$-treated cells. Conclusion: Our results demonstrate that miR-9-5p could inhibit TGF- $\beta 1$-induced HSC activation through TGFBR1 and TGFBR2. Loss of miR-9-5p
\end{abstract} is associated with its methylation status in liver fibrosis. 


\section{Introduction}

Liver fibrosis, which is caused by viral hepatitis, alcohol (non-alcoholic) steatohepatitis and several other etiologies persistent damage and stimulation, may finally result in cirrhosis and hepatocellular carcinoma [1]. Due to hepatitis B virus (HBV) infection, there are far more than 93 million HBV carriers in China [2]. HBV carriers have the potential to become liver fibrosis or even liver cirrhosis. Therefore, inhibition of fibrosis development at early stage may be an effective strategy for liver fibrosis prevention and treatment. It is well known that activation of hepatic stellate cells is a key event in liver fibrosis [3, 4]. Upon response to fibrogenic stimuli, quiescent HSCs transdifferentiate into myofibroblast-like cells, which is characterized by a-smooth muscle actin ( $\alpha$-SMA) and enhanced extracellular matrix (ECM) components. Among the profibrogenic mediators, transforming growth factor- $\beta 1$ (TGF- $\beta 1$ ) is considered as the main profibrogenic inducer [5].

MicroRNAs (miRNAs), endogenous small non-coding RNAs, have been reported to be involved in liver fibrosis [6-8]. It is known that miRNAs play a key role in repressing translation or enhancing mRNA cleavage to control gene expression by pairing with the 3'untranslated region (3'UTR) of target mRNAs [9]. For example, miR-122 regulates collagen production via targeting HSCs and suppressing P4HA1 expression [10]. miRNAs serve as key regulators in HSC activation. Recently, increasing evidence shows that down-regulation of miR-9-5p is found in liver fibrosis [11,12]. Restoration of miR-9-5p expression inhibits HSC activation through targeting MRP1/ABCC1 [11]. Therefore, miR-9-5p may serve as a suppressor in liver fibrosis. However, the underlying mechanism of the anti-fibrotic role of miR-9-5p in liver fibrosis remains largely unknown. Due to the important role of TGF $\beta$ / Smads pathway in liver fibrosis, it is still not clear whether miR-9-5p inhibits TGF $\beta /$ Smads pathway in the activation of HSCs. In addition, the underlying mechanism of loss of miR-9-5p during liver fibrosis remains unknown. In this study, we aimed to explore whether miR-9-5p promoter methylation was responsible for miR-9-5p down-regulation and the role of miR9-5p in TGF $\beta /$ Smads pathway.

\section{Materials and Methods}

\section{Human specimens}

Liver biopsy samples were obtained from patients attending the First Affiliated Hospital of Wenzhou Medical University and Ningbo Yinzhou Second Hospital from 2007.1 to 2015.12 (Table 1). 45 therapynaive patients with chronic hepatitis $\mathrm{B}(\mathrm{CHB})$, who had undergone liver biopsy for staging and grading of liver fibrosis as well as 15 healthy controls (with normal liver biochemistry, no history of liver disease or alcohol abuse and no viral hepatitis) were enrolled in the present study. Inclusion criteria were CHB defined by detectable serum HBs antigen and serum HBV DNA for more than six months. Exclusion criteria were: (i) patients aged less than 16 years, (ii) co-infection with human immunodeficiency virus (HIV), (iii) coexistence of liver injury caused by other etiologies, including hepatitis C virus (HCV) infection, drug intake, alcohol consumption and auto-immune hepatitis, (iv) severe systematic diseases, (v) pregnancy and lactation [13]. In addition, demographic and clinical information was obtained from all patients. The study was approved by the Ethics Committee of the First Affiliated Hospital of Wenzhou Medical University (Wenzhou, China), and informed consent for the use of blood samples was obtained from all participants of the study. All procedures were performed in accordance with the current international guidelines, standards on human experimentation of the Ethics Committee of the First Affiliated Hospital of Wenzhou Medical University (Wenzhou, China), and the Helsinki Declaration of 1975, revised in 1983.

\section{Liver histology}

Liver biopsy was performed using a 16-gauge Menghini needle. Each liver biopsy case was advised by physicians in care, and liver specimens at least in $2.0 \mathrm{~cm}$ in length obtained. Samples were fixed in formalin, embedded in paraffin, and stained with hematoxylin-eosin. Results were reviewed by experienced hepatopathologists [14]. In addition, at least 8-10 portal tracts in samples are required to admit patients. Histological activity index (HAI) and fibrosis stages (F0 = no fibrosis - F6 = cirrhosis) were assessed using the Ishak scoring system [15]. 


\section{Cellular Physiology Cell Physiol Biochem 2017;43:2242-2252 \begin{tabular}{ll|l} 
and Biochemistry Published onlIne: October 27, 2017 & $\begin{array}{l}\text { (c) } 2017 \text { The Author(s). Published by S. Karger AG, Basel } \\
\text { www.karger.com/cpb }\end{array}$ \\
\hline
\end{tabular} \\ Yu et al.: The Roles of miR-9-5p in HSC Activation}

Carbon tetrachloride ( $\left.\mathrm{CCl}_{4}\right)$ liver injury Table 1. Patient Characteristics model

Eight-week-old male C57BL/6J mice $(n=6)$ received intraperitoneal injection of 7 $\mu \mathrm{L} / \mathrm{g}$ of $10 \% \mathrm{CCl}_{4}$ (Sigma-Aldrich) in olive oil two times weekly for six weeks. Meanwhile, mice $(n=6)$ treated with olive oil treatment were considered as the control mice. As well as oil treatment and $\mathrm{CCl}_{4}$ treatment, mice additionally received $\mathrm{CCl}_{4}$ in combination with adenoviral vectors expressing the scrambled control (Ad-ctrl) $(\mathrm{n}=6)$ and $\mathrm{CCl}_{4}$ in combination with Ad-miR-9-5p $(n=6)$. AdmiR-9-5p $\left(1 \times 10^{9} \mathrm{pfu} / 100 \mu \mathrm{L}\right)$ was injected every two weeks by way of the tail vein for 6 weeks. Ad-miR-9-5p and Ad-ctrl were purchased from GenePharma biotechnology (Shanghai, China). The animals were provided by the Experimental Animal Center

\begin{tabular}{|c|c|c|}
\hline Parameter & CHB patients & Healthy subjects \\
\hline \multicolumn{3}{|l|}{ Epidemiology } \\
\hline Gender, $\mathrm{m} / \mathrm{f}(\%)$ & $28 / 17(62.2 / 37.8)$ & $9 / 6(60.0 / 40.0)$ \\
\hline Age, years, median (range) & $44.1(36.7-50.4)$ & $42.4(34.4-47.2)$ \\
\hline \multicolumn{3}{|l|}{ Virology } \\
\hline HBe antigen positive, $n(\%)$ & 18 & \\
\hline HBe antigen negative, $\mathrm{n}(\%)$ & 27 & \\
\hline \multicolumn{3}{|l|}{ Fibrosis stage (Ishak) } \\
\hline $\mathrm{F} 0, \mathrm{n}(\%)$ & $8(17.8 \%)$ & \\
\hline F1, n (\%) & $7(15.6 \%)$ & \\
\hline F2, n (\%) & $3(6.7 \%)$ & \\
\hline F3, n (\%) & $6(13.3 \%)$ & \\
\hline $\mathrm{F} 4, \mathrm{n}(\%)$ & $6(13.3 \%)$ & \\
\hline F5, n (\%) & $4(8.9 \%)$ & \\
\hline F6, n (\%) & $11(24.4 \%)$ & \\
\hline \multicolumn{3}{|l|}{ HAI } \\
\hline $2, \mathrm{n}(\%)$ & $7(15.5 \%)$ & \\
\hline $3, \mathrm{n}(\%)$ & $3(6.7 \%)$ & \\
\hline $4, \mathrm{n}(\%)$ & $3(6.7 \%)$ & \\
\hline $5, \mathrm{n}(\%)$ & $5(11.1 \%)$ & \\
\hline $6, n(\%)$ & $6(13.3 \%)$ & \\
\hline $7, \mathrm{n}(\%)$ & $5(11.1 \%)$ & \\
\hline $8, \mathrm{n}(\%)$ & $3(6.7 \%)$ & \\
\hline $9, \mathrm{n}(\%)$ & $5(11.1 \%)$ & \\
\hline$\geq 11, \mathrm{n}(\%)$ & $8(17.8 \%)$ & \\
\hline
\end{tabular}
of Wenzhou Medical University. The animal experimental protocol was approved by the University Animal Care and Use Committee. Mice were sacrificed under anesthesia at the end of six weeks and the livers were removed for further analysis. The liver tissues were used for Sirius Red staining.

\section{Hepatic hydroxyproline content}

Liver tissues $\left(50 \mathrm{mg}\right.$ ) were homogenized in $\mathrm{HCl}$ and hydrolyzed at $120^{\circ} \mathrm{C}$ overnight. After lysate centrifugation at $12,000 \mathrm{~g}$ for $10 \mathrm{~min}$ at $4^{\circ} \mathrm{C}$, the supernatant was evaporated to dryness under vacuum. The hepatic hydroxyproline content was assessed using the Hydroxyproline Colorimetric Assay kit (BioVision, San Francisco, CA, cat\# K555-100). Data were normalized to liver weight.

\section{Cell culture}

The human LX-2 cell strain was obtained from JENNIO Biological Technology (Guangdong, China). Cells were cultured in DMEM containing 10\% fetal bovine serum, $100 \mathrm{U} / \mathrm{ml}$ penicillin G sodium salt and $100 \mathrm{U} /$ $\mathrm{ml}$ streptomycin sulfate (Gibco, Carlsbad, CA, USA), and incubated at $37^{\circ} \mathrm{C}$ under an atmosphere of $5 \% \mathrm{CO}_{2}$. Cells were treated with $2 \mathrm{ng} / \mathrm{ml}$ TGF- $\beta 1$ (R\&D Systems, Shanghai, China) and then were transfected with 10 nM miR-9-5p mimics or inhibitor (GenePharma biotechnology, Shanghai, China) using Lipofectamine 2000 (Invitrogen, USA). Cells were harvested for RNA/miRNA isolation, and whole cell extracts were subjected to western blot analysis.

\section{Proliferation analysis}

Cell proliferation was determined by the 3-(4, 5-dimethylthiazol-2-yl)-2, 5-diphenyl-tetrazolium bromide (MTT) assays (Beyotime Biotechnology, Jiangsu, China) according to the instructions. Briefly, the cells were seeded at a density of $5 \times 10^{3}$ cells per well in 96 -well culture plates. Then, TGF- $\beta 1$-treated cells were transfected with miR-9-5p mimics or negative control. Cell lysates were prepared after their respective treatment. The cells were incubated with $0.5 \%$ MTT for $4 \mathrm{~h}$. Upon removal of the supernatant, $150 \mu \mathrm{l}$ dimethyl sulfoxide (DMSO) was added and shaken for $5 \mathrm{~min}$ until the crystals were dissolved. The optical density (OD) was determined with a microplate reader (Bio-Rad 550, USA) at $570 \mathrm{~nm}$ wavelength. In addition, HSCs were labelled with EdU for $12 \mathrm{~h}$. The HSC proliferative rate was detected using a CellLight $^{\mathrm{TM}}$ EdU in vitro Imaging Detection Kit (Guangzhou RiboBio Co., Ltd., cat\# C10310-1) according to the manufacturer's instructions.

\section{Quantitative real-time PCR ( $q R T-P C R)$}

To examine mRNA expression, total RNA was extracted from cells using TRIzol Kit (Life Technologies). Equal amounts of total RNA were reverse-transcribed to cDNA using the ReverTra Ace qPCR RT Kit (Toyobo, Osaka, Japan). Gene expression was measured by real-time PCR using SYBR Green real-time PCR Master 


\section{Cellular Physiology Cell Physiol Biochem 2017;43:2242-2252 and Biochemistry Published onine $\begin{aligned} & \text { DOI: 10.1159/000484303 } 2017 \text { The Author(s). Published by S. Karger AG, Basel } \\ & \text { www.karger.com/cpb }\end{aligned}$}

Yu et al.: The Roles of miR-9-5p in HSC Activation

Mix (Toyobo, Osaka, Japan). The primers of alpha-1 (I) collagen (Col1A1), $\alpha$-SMA, GAPDH and U6 were designed as described previously $[6,16]$. The primers used for type I TGF- $\beta$ receptor (TGFBR1) were $5^{\prime}$-AGGGAAAGTCTGTCTAGCTGC- $3^{\prime}$ and $5^{\prime}$-ACTAC CTTCGCCTTCCTAGA- ${ }^{\prime}$. The primers used for type II TGF- $\beta$ receptor (TGFBR2) were $5^{\prime}$-GCAGCGCTGAGTTGAAGTTG- $3^{\prime}$ and $5^{\prime}$-GAGGGAAGCTGCACAGGA G- $3^{\prime}$. To examine miRNA expression, total RNA was extracted from liver tissues and cells using a miRNeasy Mini Kit (Qiagen, Valencia, CA, USA). miR-9-5p expression was detected using the TaqMan MicroRNA Assay (Applied Biosystems, Foster City, CA). The GAPDH and U6 snRNA levels were used to normalize the relative abundance of mRNAs and miRNAs, respectively.

\section{Western blot analysis}

To obtain total protein, HSCs were lysed with ice-cold lysis buffer (50 mM Tris-HCl, pH 7.4, $100 \mathrm{mM}$ 2-Mercaptoethanol, 2\% w/v SDS, $10 \%$ glycerol). Cytoplasmic and nuclear expression of p-Smad2 was also evaluated by western blot. Cytoplasmic and nuclear extracts were prepared according to the manufacturer's instruction (Beyotime Biotechnology, Jiangsu, China) [17]. Protein samples were quantified and separated by SDS-PAGE. Then, western blot assay was performed as described previously [18].

\section{Northern blot}

RNA was separated on a $12 \%(\mathrm{w} / \mathrm{v})$ polyacrylamide gel and transferred to Hybond-NX membrane (Amersham Biosciences). The blot was hybridised in ULTRAHyb-Oligo buffer (Life Technologies) with a gATP-labelled probe complementary to the miRNA at $37^{\circ} \mathrm{C}$ overnight. Membranes were exposed to a Kodak Phosphor Screen SD230 and scanned on a Molecular Imager FX reader (Bio-Rad) for quantification. 28S and $18 \mathrm{~S}$ RNAs were set as the loading controls.

\section{Methylation analysis}

miR-9-5p CpG island was searched in UCSC Genome Browser. About $0.5 \mu \mathrm{g}$ genomic DNA was treated with sodium bisulphite and subjected to PCR. The hsa-miR-9-5p primers for PCR were 5'-GTATTTGGAATTTAGGGTTTTGG-3' and 5'-CCCAAAATTATAAAAATAACC-3'. The bisulphite-sequencing analysis was carried out as described previously [19].

\section{Luciferase activity assay}

LX-2 cells were co-transfected with either luciferase reporter plasmid harboring wild-type TGFBR1 3'UTR (pmirGLO-TGFBR1-wt) or mutant TGFBR1 3'UTR (pmirGLO-TGFBR1-mut) together with miR-9$5 p$ mimics or miR-NC. The 3'UTR of TGFBR1 was cloned downstream of the firefly luciferase gene. If the interaction between miR-9-5p and the 3'UTR of TGFBR1 mRNA was existed, the activity of firefly luciferase was decreased. The same analysis was performed in pmirGLO-TGFBR2-wt and pmirGLO-TGFBR2-mut. Approximately $48 \mathrm{~h}$ after transfection, the cells were harvested and luciferase activity was determined by a luminometer using a Dual-Luciferase Reporter Assay System (Promega, Madison, WI, USA).

\section{Statistical analysis}

Data from at least three independent experiments were expressed as the mean \pm SD. The MannWhitney test or Kruskal-Wallis test was performed to determine the significance of miR-9-5p in CHB patients. Comparisons between two groups and multiple groups were made using Student's $t$-test and one-way analysis of variance, respectively. $P<0.05$ was considered significant. All statistical analyses were performed with SPSS software (version 13; SPSS, Chicago, IL).

\section{Results}

\section{Down-regulation of liver miR-9-5p expression in liver fibrosis}

A total of 60 subjects were recruited, including 45 CHB patients and 15 healthy controls (Table 1). In this study, there were no significant differences in age between CHB patients and healthy controls $(P=.650)$. Moreover, no significant difference was observed in sex distribution $\left(P=.305, \chi^{2}\right.$ test). To investigate whether miR-9-5p is reduced in CHB patients, qRT-PCR analysis was performed to detect miR-9-5p expression in liver tissues from CHB patients as well as healthy controls. Compared with healthy controls, miR-9-5p was reduced in CHB patients (Fig.1A). According to the fibrosis scores, all CHB patients were divided into 


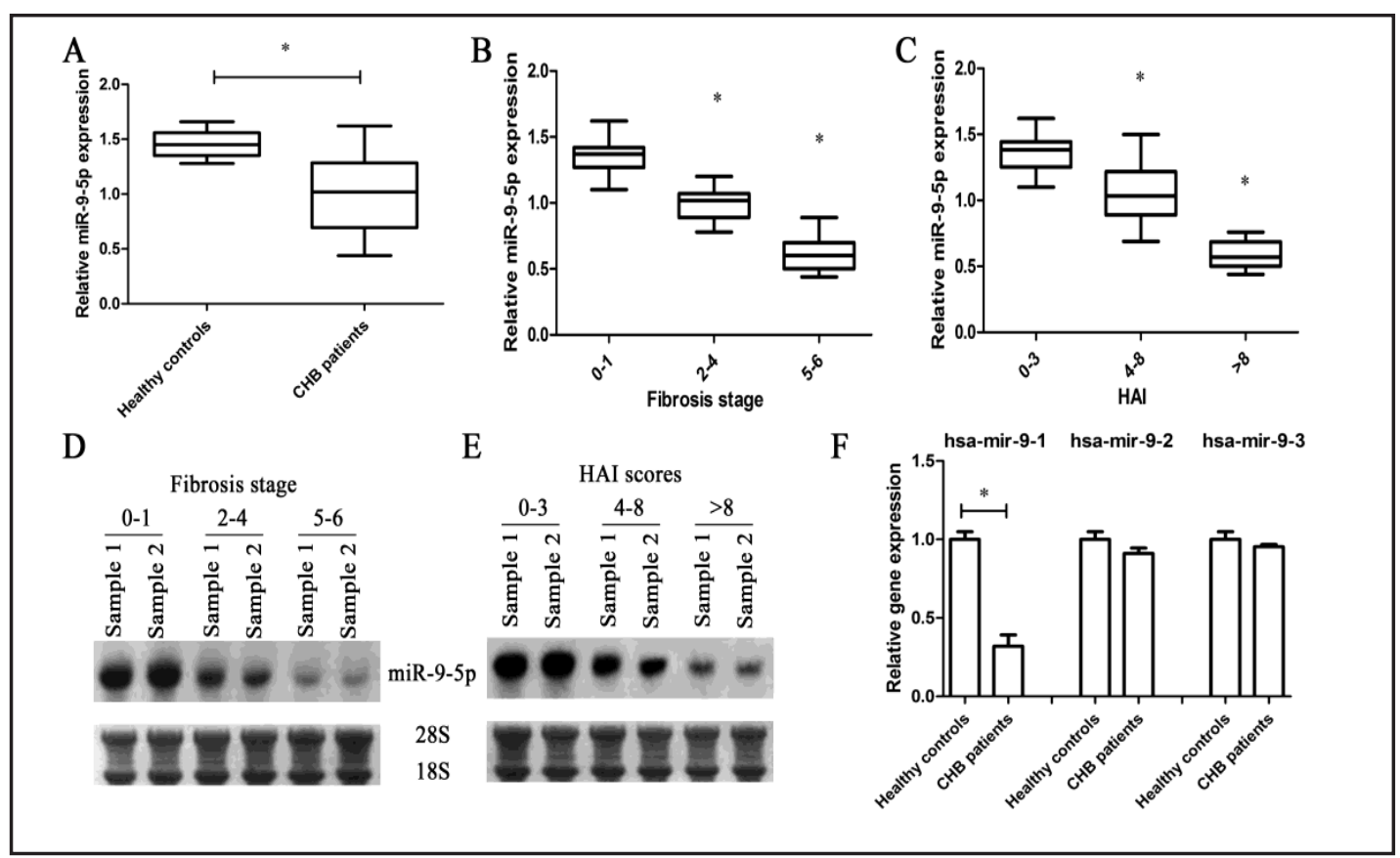

Fig. 1. Reduced liver miR-9-5p levels in CHB patients. (A) miR-9-5p expression in CHB patients and healthy controls. miR-9-5p expression in patients with different fibrosis scores (B) or different HAI scores (C). Using Northern bolt analysis, lower miR-9-5p expression was confirmed in CHB patients with higher fibrosis scores (D) or higher HAI scores (E). (F) The relative expressions of hsa-mir-9-1, hsa-mir-9-2 and hsamir-9-3 in healthy controls and CHB patients. Each value is the mean \pm SD of three experiments. ${ }^{*} \mathrm{P}<0.05$ compared with the control.

three groups including low-score group (0-1), medium-score group (2-4) and high-score group (5-6). Then, the association between miR-9-5p and fibrosis stages was explored. There was a lower miR-9-5p expression in CHB patients with higher fibrosis scores (Fig.1B). Next, the association between miR-9-5p and HAI scores was investigated. With the increasing HAI scores, there was a significant reduction in miR-9-5p (Fig.1C). Using Northern blot analysis, reduced miR-9-5p was further confirmed in CHB patients with higher fibrosis scores or HAI scores (Fig.1D and Fig.1E). Next, miR-9-5p expression was detected in TGF- $\beta 1$-treated LX-2 cells. TGF- $\beta 1$ induced a decrease in miR-9-5p expression in a dose-dependent manner, ranging from concentrations of 0 to $5 \mathrm{ng} / \mathrm{ml}$ (Fig.2A). Combined with these, our data indicate that miR-9-5p is down-regulated in liver fibrosis.

\section{miR-9-5p inhibits liver fibrosis in vitro and in vivo}

We next explored whether restoration of miR-9-5p contributed to the suppression of TGF- $\beta 1$-induced cell proliferation, HSC transdifferentiation and ECM production. MTT assays showed that TGF- $\beta 1$-caused cell proliferation was blocked down by miR-9-5p mimics, which was further confirmed by Edu assays (Fig.2B and Fig.2J). $\alpha$-SMA, a key marker widely accepted for HSC transdifferentiation, is often expressed at high levels in activated HSCs [20]. The mRNA expression of $\alpha$-SMA induced by TGF- $\beta 1$ was inhibited by miR-9-5p overexpression (Fig.2C). Similarly, increased $\alpha$-SMA protein caused by TGF- $\beta 1$ was reversed by miR-9-5p (Fig.2E). The effect of miR-9-5p over-expression on collagen expression was also explored. The mRNA expression of Col1A1 caused by TGF- $\beta 1$ was suppressed by miR-9-5p (Fig.2D). Also, type I collagen induced by TGF- $\beta 1$ was blocked down by miR-9-5p (Fig.2F). Taken together, miR-9-5p inhibits TGF- $\beta 1$-induced HSC activation. To confirm the role of miR9-5p in liver fibrosis in vivo, the effects of miR-9-5p over-expression on liver fibrosis were explored. As shown by Sirius Red staining, $\mathrm{CCl}_{4}$-induced collagen expression was inhibited by miR-9-5p over-expression (Fig.2G and Fig.2H). In lined with it, liver hydroxyproline analysis 


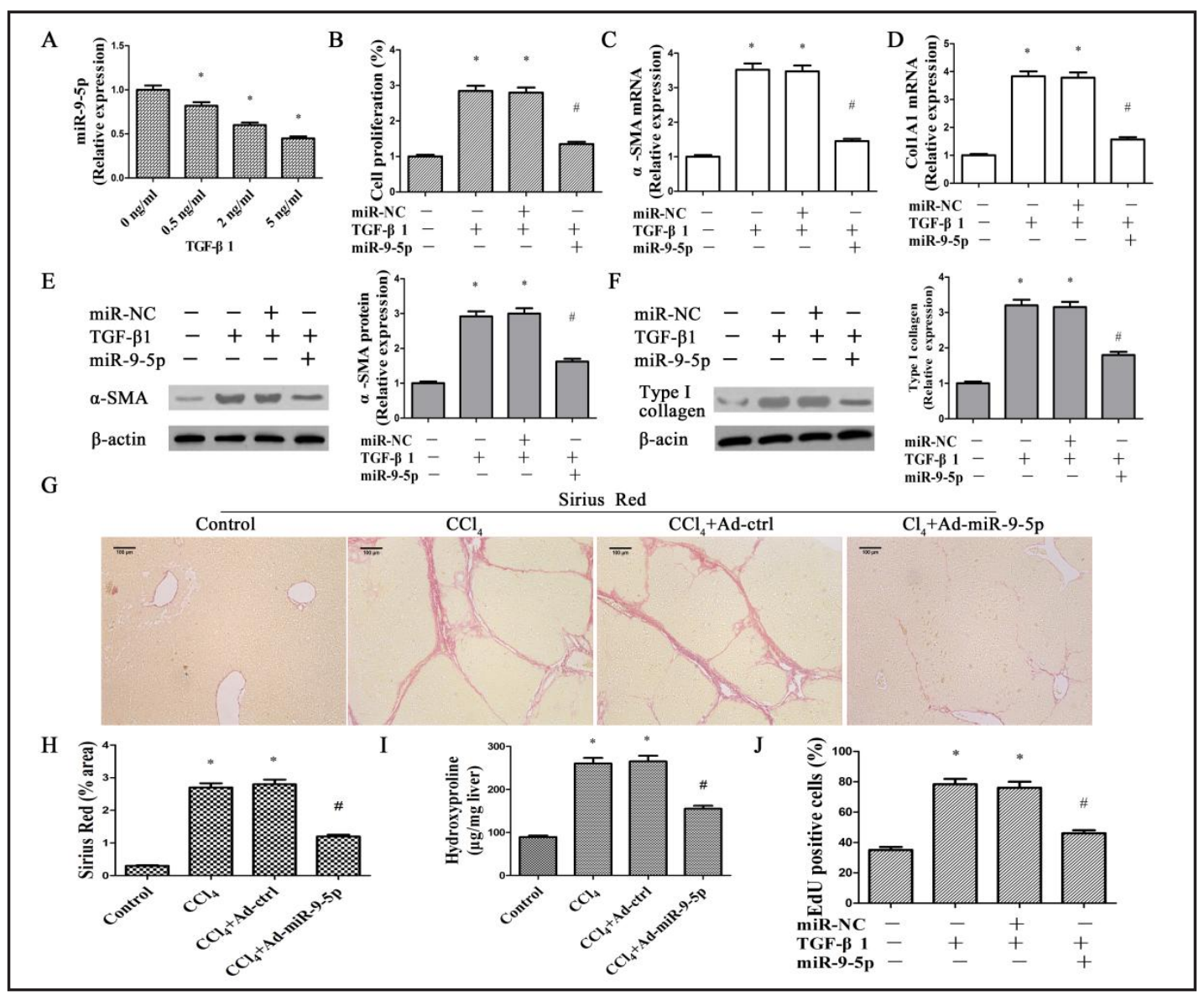

Fig. 2. Effects of miR-9-5p on HSC activation in vitro and in vivo. LX- 2 cells were treated with TGF- $\beta 1$ for $24 \mathrm{~h}$ and then transfected with miR-9-5p mimics for $24 \mathrm{~h}$. (A) miR-9-5p expression. (B) Cell proliferation detected by MTT. (C) $\alpha$-SMA mRNA. (D) Col1A1 mRNA. (E) $\alpha$-SMA protein. (F) Type I collagen. (G and H) Collagen deposits were analyzed by Sirius Red staining. Scale bars, $100 \mu \mathrm{m}$. (I) Hydroxyproline expression. (J) Cell proliferation detected by EdU. Each value is the mean \pm SD of three experiments. ${ }^{*} \mathrm{P}<0.05$ compared with the control and ${ }^{\#} \mathrm{P}<0.05$ compared with $\mathrm{TGF}-\beta 1$ or $\mathrm{CCl}_{4}$ group.

showed that $\mathrm{CCl}_{4}$-induced hydroxyproline was reversed by miR-9-5p over-expression (Fig.2I). Our data suggest that miR-9-5p could ameliorate liver fibrosis.

\section{miR-9-5p targets TGFBR1 and TGFBR2}

The aberrant activated TGF- $\beta /$ Smad signal pathway has been reported to contribute to the activation of HSCs [21]. TGF- $\beta 1$ induces HSC activation via $p$-Smad2/3 [5]. At the present study, miR-9-5p suppressed TGF- $\beta 1$-induced $p$-Smad2 level while miR-9-5p inhibitor promoted $p$-Smad2 level in TGF- $\beta 1$-treated cells, suggesting that miR-9-5p could inhibit TGF- $\beta /$ Smads signaling (Fig.3A and Fig.3B). Consistent with it, TGF- $\beta 1$-increased $\mathrm{p}$-Smad2 level in cytoplasm and nuclear was inhibited by miR-9-5p mimics, which was enhanced by miR-9-5p inhibitor (Fig.3A and Fig.3B). To exclude nuclear contamination in the cytoplasmatic fraction, Lamin A (a marker for the nuclear fraction) was detected in the cytoplasmatic fraction. Also, HSP90 (a marker for the cytoplasmatic fraction) was detected in the nuclear fraction. It was confirmed that LaminA was not found in the cytoplasmatic fraction and HSP90 was not found in the nuclear fraction. Smad7, an inhibitor of TGF- $\beta$ / Smad pathway, is often decreased by TGF- $\beta 1$. Interestingly, reduced Smad7 in TGF- $\beta 1$ group was restored by miR-9-5p, which was further reduced by miR-9-5p inhibitor. Combined with these, we demonstrated that miR-9-5p could inhibit TGF- $\beta /$ Smad pathway. To further

\section{KARGER}




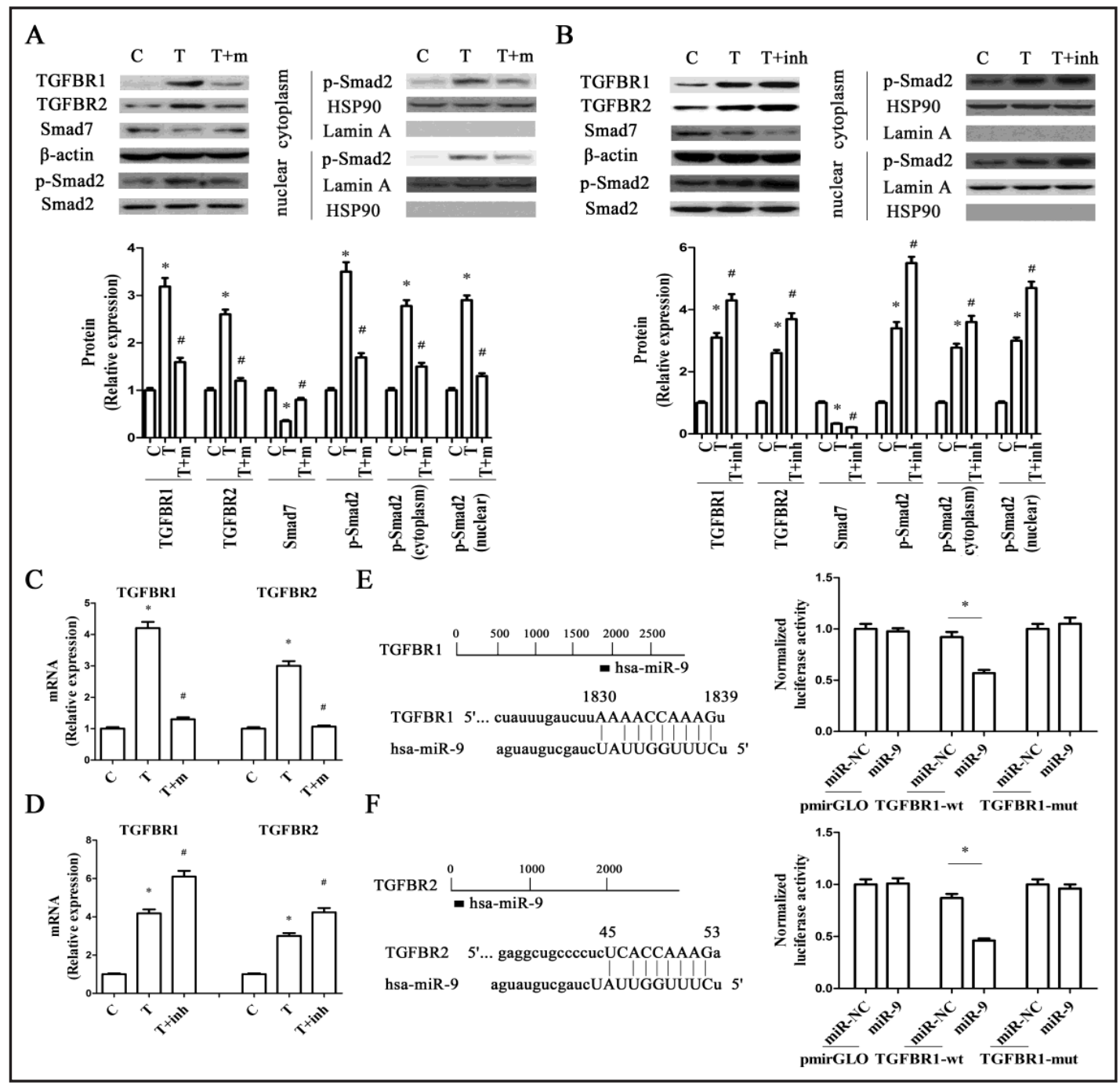

Fig. 3. Over-expression of miR-9-5p inhibited TGF- $\beta 1 /$ Smads pathway through TGFBR1 and TGFBR2. LX-2 cells were treated with TGF- $\beta 1$ for 15 min and then transfected with miR-9-5p mimics or its inhibitor for $24 \mathrm{~h}$. (A and B) The protein expressions of TGFBR1, TGFBR2, p-Smad2 and Smad7, and the protein levels of p-Smad2 in cytoplasm and nuclear. (C and D) The mRNA expressions of TGFBR1 and TGFBR2. (E) miR-9-5pbinding sites in the 3'UTR of TGFBR1 mRNA based on microRNA.org software. Relative luciferase activities of luciferase reporters bearing wild-type or mutant TGFBR1 were analyzed $48 \mathrm{hr}$ following transfection with the indicated miR-9-5p mimics or miR-NC. (F) miR-9-5p-binding sites in the $3^{\prime}$ UTR of TGFBR2 mRNA based on microRNA.org software. Relative luciferase activities of luciferase reporters bearing wild-type or mutant TGFBR2 were analyzed $48 \mathrm{hr}$ following transfection with the indicated miR-9-5p mimics or miRNC. Each value is the mean $\pm \mathrm{SD}$ of three experiments. ${ }^{*} \mathrm{P}<0.05$ compared with the control and ${ }^{*} \mathrm{P}<0.05$ compared with the TGF- $\beta 1$ group. C: control group. T: TGF- $\beta 1$ group. T+m: TGF- $\beta 1+$ miR- $9-5 p$ mimics group. T+inh: TGF- $\beta 1+$ miR-9-5p inhibitor group.

investigate the mechanism by which miR-9-5p suppresses TGF- $\beta 1$-induced HSC activation, bioinformatic analysis (microRNA.org) was employed to identify the potential targets of miR-9-5p. Among the miR-9-5p target genes, TGFBR1 and TGFBR2 were chosen to the next experiments because of their roles in TGF- $\beta$ /Smads signaling. To investigate the effects of miR-9-5p on TGFBR1 and TGFBR2, the mRNA and protein expressions of TGFBR1 and TGFBR2 were examined in TGF- $\beta 1$-treated LX-2 cells transfected with miR-9-5p mimics or its inhibitor. Our results showed that increased protein levels of TGFBR1 and TGFBR2 induced 
A

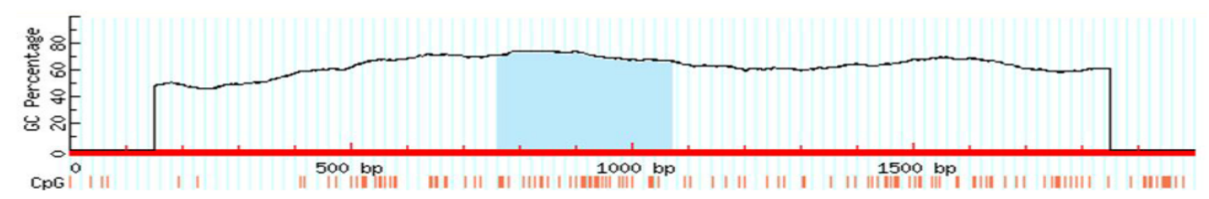

B

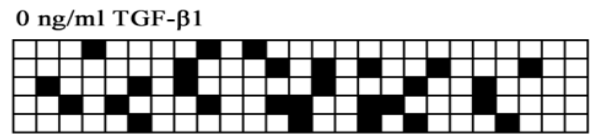

$0.5 \mathrm{ng} / \mathrm{ml}$ TGF- $\beta 1$

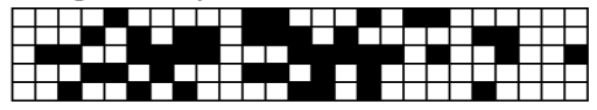

$2.0 \mathrm{ng} / \mathrm{ml}$ TGF- $\beta 1$

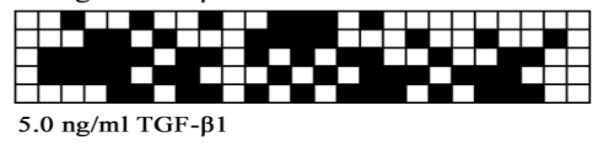

$5.0 \mathrm{ng} / \mathrm{ml}$ TGF- $\beta 1$

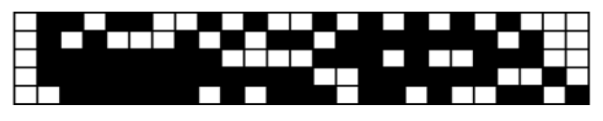

C Healthy controls

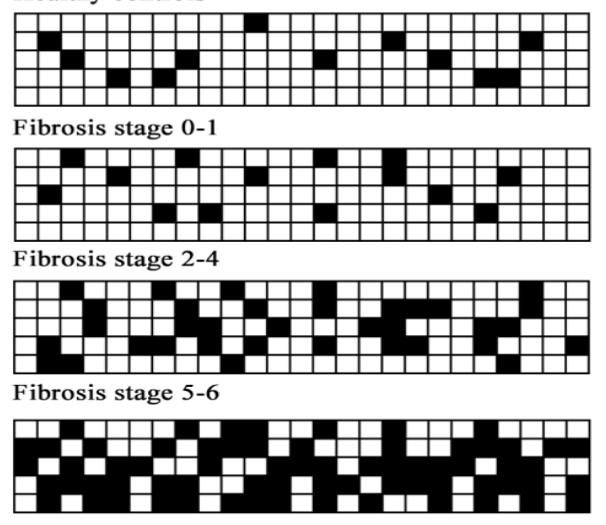

Fig. 4. The promoter methylation status of miR-9-5p in TGF- $\beta 1$-treated cells and in the livers of CHB patients. (A) Schematic representation of CpG sites in the promoter region of hsa-miR-9-1. Each vertical bar represents the presence of a CpG dinucleotide. (B) The methylation of miR-9-5p was detected by bisulfide sequencing in LX-2 cells treated with TGF- $\beta 1(0,0.5,2$ and $5 \mathrm{ng} / \mathrm{ml})$. Each row of boxes represents the sequence analysis of an individual clone from the PCR products (white box, unmethylated CpG site; black box, methylated CpG site). (C) The methylation of miR-9-5p was detected by bisulfide sequencing in CHB patients. Each row of boxes represents the sequence analysis of an individual clone from the PCR products (white box, unmethylated CpG site; black box, methylated CpG site).

by TGF- $\beta 1$ were inhibited by miR-9-5p over-expression, which were enhanced by miR-9-5p inhibitor (Fig.3A and Fig.3B). In lined with it, TGF- $\beta 1$-induced the mRNA levels of TGFBR1 and TGFBR2 were inhibited by miR-9-5p, which was further increased by miR-9-5p inhibitor (Fig.3C and Fig.3D). Next, we cloned the 3'UTR target sequence of TGFBR1 mRNA into the pmirGLO plasmid to confirm whether miR-9-5p could directly regulate TGFBR1 expression via the predicted binding site (Fig.3E). It was found that miR-9-5p mimics led to a significant reduction in luciferase activities of pmirGLO-TGFBR1-wt with no effect on pmirGLO-TGFBR1mut (Fig.3E). Whether TGFBR2 was a target of miR-9-5p was also explored. As shown in Fig.3F, miR-9-5p significantly reduced luciferase activities of pmirGLO-TGFBR2-wt, whereas miR-9-5p had no effect on luciferase activities of pmirGLO-TGFBR2-mut. Taken together, miR-9-5p inhibits TGF- $\beta$ /Smads signaling, at least in part, through targeting of TGFBR1 and TGFBR2.

\section{Promoter methylation status of miR-9-5p in TGF- $\beta 1$-treated HSCs and CHB patients}

Due to hsa-miR-9-5p is encoded by three distinct genomic loci, miR-9 family contains three precursors (i.e., hsa-mir-9-1, hsa-mir-9-2, and hsa-mir-9-3). Compared with healthy controls, there was a significant reduction in hsa-mir-9-1 level in CHB patients (Fig.1F). Meanwhile, there was not obvious reduction in hsa-mir-9-2 and hsa-mir-9-3 expression. Therefore, hsa-mir-9-1 was chosen for the next experiments. Increasing evidence suggests that miRNAs down-regulation in some malignant cells may be caused by aberrant promoter methylation [22]. Previously, it has been reported that aberrant hypermethylation of miR9-5p was found in hepatocellular carcinoma (HCC) [23]. Next, we explored whether the promoter of miR-9-5p was aberrantly methylated in liver fibrosis. Using bisulfite-sequencing analysis, we examined the methylation status of the $\mathrm{CpG}$ island located hsa-mir-9-1 promoter (Fig.4A). Firstly, miR-9-5p methlyation rate was detected in cells with different 
concentration of TGF- $\beta 1$. The mean frequency of miR-9-5p methylation was $22.4 \%$ in LX-2 cells without TGF- $\beta 1$ (Fig.4B). In comparison with the control, there was a significant increase in methylation frequency in TGF- $\beta 1$-treated cells, with the highest methylation frequency at the concentration of $5 \mathrm{ng} / \mathrm{ml}$ (Fig.4B). With the increase of TGF- $\beta 1$ concentration, there was a significant reduction in miR-9-5p in a dose-dependent manner. There may be a negative correlation between miR-9-5p expression and its promoter methylation. Then, the relation between miR-9-5p expression and its promoter methylation was further determined in human liver tissues. The mean frequency of miR-9-5p methylation was $9.6 \%$ in healthy controls while it was $12.0 \%$ in CHB patients with low fibrosis scores, indicating that there was no obvious change of miR-9-5p methylation between healthy controls and patients with low fibrosis (Fig.4C). Compared with CHB patients with low fibrosis scores, miR-9-5p methylation was increased in CHB patients with higher fibrosis scores (Fig.4C). As shown in Fig.1B, with the increase of fibrosis scores, miR-9-5p expression was reduced. Therefore, the relation between miR-9-5p expression and promoter methylation was confirmed. In sum, our data suggest that miR-9-5p expression is associated with its promoter methylation in liver fibrosis.

\section{Discussion}

A growing body of evidence suggests that miR-9-5p is involved in various human diseases [24, 25]. For example, Moazzeni et al. reported that miR-9-5p, functions as a tumor suppressor miRNA, can induce apoptosis and inhibit proliferation in breast cancer cells [25]. In organ fibrosis, miR-9-5p may also act as a negative regulator. For instance, miR-9-5p regulates cardiac fibrosis by targeting PDGFR- $\beta$ in rats [26]. A recent study has demonstrated that miR-9-5p has a protective role in the fibrogenic transformation of human dermal fibroblasts [27]. In liver fibrosis, a recent study shows that miR-9-5p not only inhibits HSC proliferation and activation in vitro but also inhibited $\mathrm{CCl}_{4}$-induced liver fibrosis in mice [11]. In this study, miR-9-5p expression was down-regulated in CHB patients as well as in TGF- $\beta 1$-activated HSCs, which was consistent with the previous reports in organ fibrosis $[11,28]$. Notably, miR-9-5p inhibited TGF- $\beta 1$-caused cell proliferation, $\alpha$-SMA and collagen expression, suggesting its anti-fibrotic role in HSC activation. Moreover, miR-9-5p ameliorates $\mathrm{CCl}_{4}$-caused liver fibrosis. We demonstrate that miR-9-5p can inhibit TGF- $\beta /$ Smads signaling in liver fibrosis.

Aberrant activated TGF- $\beta$ /Smads signaling is involved in liver fibrosis and contributes to the progression of liver fibrosis [29]. Suppression of TGF- $\beta$ expression or the activity of TGF- $\beta$ /Smads signaling by many therapeutic strategies shows anti-fibrotic effects in fibrotic diseases [30]. The TGF- $\beta$ family contains three closely related isoforms (i.e., TGF- $\beta 1$, TGF- $\beta 2$, and TGF- $\beta 3$ ) and TGF- $\beta 1$ is recognized as the main profibrogenic mediator [31]. Generally, TGF- $\beta 1$ binds to the TGFBR1, and then recruits and activates the TGFBR2 [32]. Once TGFBR1 was activated, it will phosphorylate $\operatorname{Smad} 2 / 3$ and then initiate TGF- $\beta /$ Smads signaling. At the present study, we found that miR-9-5p inhibited p-Smad2 level induced by TGF- $\beta 1$ and restored TGF- $\beta 1$-suppressed Smad7, suggesting that miR-9-5p could lead to the suppression of TGF- $\beta /$ Smads signaling. By contrast, miR-9-5p inhibitor contributed to an increase in the activity of TGF- $\beta /$ Smads signaling. Interestingly, miR-9-5p over-expression resulted in the suppression of TGFBR1 and TGFBR2. Further studies confirmed that TGFBR1 and TGFBR2 were targets of miR-9-5p. Our results demonstrated that miR-9-5p inhibits TGF- $\beta /$ Smads signaling, at least in part, via TGFBR1 and TGFBR2.

An increasing number of studies suggest that epigenetic changes play a key role in various human diseases, especially in cancers [33, 34]. DNA methylation, as a type of epigenetic modifications in mammals, has been reported to be associated with normal transcriptional regulation. For example, Bian et al. found that PTEN hypermethylation confers hepatic stellate cell activation and liver fibrogenesis [35]. Recently, DNA methylation is involved in the down-regulation of miRNA expression. Previously, we found that reduced miR-378a was associated with its promoter methylation in liver fibrosis [36]. In this study, with the increase 
of TGF- $\beta 1$ dose, miR-9-5p expression was gradually reduced, whereas miR-9-5p methylation was enhanced in a dose-dependent manner. Our data suggest a negative correlation between miR-9-5p expression and promoter methylation. This correlation was further confirmed in CHB patients with different fibrosis stages. Therefore, promoter methylation is required for miR-9-5p down-regulation in liver fibrosis. Our results additionally indicated that miR-9$5 p$ was associated with fibrosis stages, suggesting that miR-9-5p may serve as a potential biomarker in patients with liver fibrosis. Moreover, miR-9-5p expression was associated with liver inflammatory activity markers such as HAI scores, indicating that miR-9-5p may represent a marker of necroinflammation in CHB patients. However, the sample size is relatively small and large samples are needed to the further validations of this marker. In addition, the detection of serum miRNAs in peripheral blood has provided a novel approach for noninvasive clinical diagnosis. The clinical significance of serum miR-9-5p in liver fibrosis needs to be explored in future.

In conclusion, our results demonstrated that miR-9-5p could inhibit HSC proliferation and activation induced by TGF- $\beta 1$ via targeting TGFBR1 and TGFBR2. In liver fibrosis, reduced miR-9-5p expression is associated with promoter methylation status.

\section{Acknowledgements}

The project was supported by the National Natural Science Foundation of China (No. 81500458/H0317), Zhejiang Provincial Natural Science Foundation of China (No. LY16H030012) and Wenzhou Municipal Science and technology Bureau (No. Y20150091).

\section{Disclosure Statement}

None.

\section{References}

1 Ogawa T, Enomoto M, Fujii H, Sekiya Y, Yoshizato K, Ikeda K, Kawada N: MicroRNA-221/222 upregulation indicates the activation of stellate cells and the progression of liver fibrosis. Gut 2012;61:1600-1609.

2 Liu X, Wan X, Li Z, Lin C, Zhan Y, Lu X: Golgi protein 73(GP73), a useful serum marker in liver diseases. Clin Chem Lab Med 2011;49:1311-1316.

-3 Yu F, Fan X, Chen B, Dong P, Zheng J: Activation of Hepatic Stellate Cells is Inhibited by microRNA-378a-3p via Wnt10a. Cell Physiol Biochem 2016;39:2409-2420.

4 Li G, Li J, Li C, Qi H, Dong P, Zheng J, Yu F: MicroRNA-125a-5p Contributes to Hepatic Stellate Cell Activation through Targeting FIH1. Cell Physiol Biochem 2016;38:1544-1552.

5 Dooley S, Delvoux B, Streckert M, Bonzel L, Stopa M, ten Dijke P, Gressner AM: Transforming growth factor beta signal transduction in hepatic stellate cells via Smad2/3 phosphorylation, a pathway that is abrogated during in vitro progression to myofibroblasts. TGFbeta signal transduction during transdifferentiation of hepatic stellate cells. FEBS Lett 2001;502:4-10.

6 Zheng J, Wu C, Lin Z, Guo Y, Shi L, Dong P, Lu Z, Gao S, Liao Y, Chen B, Yu F: Curcumin up-regulates phosphatase and tensin homologue deleted on chromosome 10 through microRNA-mediated control of DNA methylation--a novel mechanism suppressing liver fibrosis. FEBS J 2014;281:88-103.

7 Huang CF, Sun CC, Zhao F, Zhang YD, Li DJ: miR-33a levels in hepatic and serum after chronic HBV-induced fibrosis. J Gastroenterol 2015;50:480-490.

8 Yu F, Lu Z, Chen B, Wu X, Dong P, Zheng J: Salvianolic acid B-induced microRNA-152 inhibits liver fibrosis by attenuating DNMT1-mediated Patched1 methylation. J Cell Mol Med 2015;19:2617-2632.

-9 Bartel DP: MicroRNAs: genomics, biogenesis, mechanism, and function. Cell 2004;116:281-297.

10 Li J, Ghazwani M, Zhang Y, Lu J, Fan J, Gandhi CR, Li S: miR-122 regulates collagen production via targeting hepatic stellate cells and suppressing P4HA1 expression. J Hepatol 2013;58:522-528.

11 Sun J, Zhang H, Li L, Yu L, Fu L: MicroRNA-9 limits hepatic fibrosis by suppressing the activation and proliferation of hepatic stellate cells by directly targeting MRP1/ABCC1. Oncol Rep 2017;37:1698-1706.

12 Li SG, Zhou J, Zhong JH, Cao HJ, Zhu L, Liu J, Hu HJ, Lv B: Effects of miR-9 and tetramethylpyrazine on activation of hepatic stellate cells. Biol Pharm Bull 2015;38:396-401. 


\section{Cellular Physiology Cell Physiol Biochem 2017;43:2242-2252 \begin{tabular}{l|l|l} 
and Biochemistry Published onlIne: October 27, 2017 & $\begin{array}{l}\text { (c) } 2017 \text { The Author(s). Published by S. Karger AG, Basel } \\
\text { www.karger.com/cpb }\end{array}$ \\
\hline
\end{tabular}}

Yu et al.: The Roles of miR-9-5p in HSC Activation

13 Huang C, Zheng JM, Cheng Q, Yu KK, Ling QX, Chen MQ, Li N: Serum microRNA-29 levels correlate with disease progression in patients with chronic hepatitis B virus infection. J Dig Dis 2014;15:614-621.

14 Yu F, Zhou G, Li G, Chen B, Dong P, Zheng J: Serum miR-181b Is Correlated with Hepatitis B Virus Replication and Disease Progression in Chronic Hepatitis B Patients. Dig Dis Sci 2015;60:2346-2352.

15 Ishak K, Baptista A, Bianchi L, Callea F, De Groote J, Gudat F, Denk H, Desmet V, Korb G, MacSween RN, et al.: Histological grading and staging of chronic hepatitis. J Hepatol 1995;22:696-699.

16 Zheng J, Lin Z, Dong P, Lu Z, Gao S, Chen X, Wu C, Yu F: Activation of hepatic stellate cells is suppressed by microRNA-150. Int J Mol Med 2013;32:17-24.

17 Li A, Zhang J, Zhang X, Wang J, Wang S, Xiao X, Wang R, Li P, Wang Y: Angiotensin II induces connective tissue growth factor expression in human hepatic stellate cells by a transforming growth factor betaindependent mechanism. Sci Rep 2017;7:7841.

18 Yu F, Lu Z, Huang K, Wang X, Xu Z, Chen B, Dong P, Zheng J: MicroRNA-17-5p-activated Wnt/beta-catenin pathway contributes to the progression of liver fibrosis. Oncotarget 2016;7:81-93.

19 Ji W, Yang L, Yu L, Yuan J, Hu D, Zhang W, Yang J, Pang Y, Li W, Lu J, Fu J, Chen J, Lin Z, Chen W, Zhuang Z: Epigenetic silencing of 06-methylguanine DNA methyltransferase gene in NiS-transformed cells. Carcinogenesis 2008;29:1267-1275.

20 Li ZJ, Ou-Yang PH, Han XP: Profibrotic effect of miR-33a with Akt activation in hepatic stellate cells. Cell Signal 2014;26:141-148.

21 Liu X, Hu H, Yin JQ: Therapeutic strategies against TGF-beta signaling pathway in hepatic fibrosis. Liver Int 2006;26:8-22.

22 Lehmann U, Hasemeier B, Romermann D, Muller M, Langer F, Kreipe H: [Epigenetic inactivation of microRNA genes in mammary carcinoma]. Verh Dtsch Ges Pathol 2007;91:214-220.

-23 Trankenschuh W, Puls F, Christgen M, Albat C, Heim A, Poczkaj J, Fleming P, Kreipe H, Lehmann U: Frequent and distinct aberrations of DNA methylation patterns in fibrolamellar carcinoma of the liver. PLoS One 2010;5:e13688.

24 He L, Zhang L, Wang M, Wang W: miR-9 functions as a tumor inhibitor of cell proliferation in epithelial ovarian cancer through targeting the SDF-1/CXCR4 pathway. Exp Ther Med 2017;13:1203-1208.

25 Moazzeni H, Najafi A, Khani M: Identification of direct target genes of miR-7, miR-9, miR-96, and miR-182 in the human breast cancer cell lines MCF-7 and MDA-MB-231. Mol Cell Probes 2017;34:45-52.

26 Wang L, Ma L, Fan H, Yang Z, Li L, Wang H: MicroRNA-9 regulates cardiac fibrosis by targeting PDGFR-beta in rats. J Physiol Biochem 2016;72:213-223.

27 Miguel V, Busnadiego 0, Fierro-Fernandez M, Lamas S: Protective role for miR-9-5p in the fibrogenic transformation of human dermal fibroblasts. Fibrogenesis Tissue Repair 2016;9:7.

28 Ji J, Zhang J, Huang G, Qian J, Wang X, Mei S: Over-expressed microRNA-27a and 27b influence fat accumulation and cell proliferation during rat hepatic stellate cell activation. FEBS Lett 2009;583:759-766.

29 He Y, Huang C, Sun X, Long XR, Lv XW, Li J: MicroRNA-146a modulates TGF-beta1-induced hepatic stellate cell proliferation by targeting SMAD4. Cell Signal 2012;24:1923-1930.

-30 Rosenbloom J, Castro SV, Jimenez SA: Narrative review: fibrotic diseases: cellular and molecular mechanisms and novel therapies. Ann Intern Med 2010;152:159-166.

-31 Gressner AM, Weiskirchen R: Modern pathogenetic concepts of liver fibrosis suggest stellate cells and TGFbeta as major players and therapeutic targets. J Cell Mol Med 2006;10:76-99.

-32 Tu X, Zhang H, Zhang J, Zhao S, Zheng X, Zhang Z, Zhu J, Chen J, Dong L, Zang Y: MicroRNA-101 suppresses liver fibrosis by targeting the TGFbeta signalling pathway. J Pathol 2014;234:46-59.

-33 Datta J, Kutay H, Nasser MW, Nuovo GJ, Wang B, Majumder S, Liu CG, Volinia S, Croce CM, Schmittgen TD, Ghoshal K, Jacob ST: Methylation mediated silencing of MicroRNA-1 gene and its role in hepatocellular carcinogenesis. Cancer Res 2008;68:5049-5058.

-34 Garzon R, Liu S, Fabbri M, Liu Z, Heaphy CE, Callegari E, Schwind S, Pang J, Yu J, Muthusamy N, Havelange V, Volinia S, Blum W, Rush LJ, Perrotti D, Andreeff M, Bloomfield CD, Byrd JC, Chan K, Wu LC, Croce CM, Marcucci G: MicroRNA-29b induces global DNA hypomethylation and tumor suppressor gene reexpression in acute myeloid leukemia by targeting directly DNMT3A and 3B and indirectly DNMT1. Blood 2009;113:6411-6418.

35 Bian EB, Huang C, Ma TT, Tao H, Zhang H, Cheng C, Lv XW, Li J: DNMT1-mediated PTEN hypermethylation confers hepatic stellate cell activation and liver fibrogenesis in rats. Toxicol Appl Pharmacol 2012;264:1322.

-36 Yu F, Yang J, Huang K, Pan X, Chen B, Dong P, Zheng J: The Epigenetically-Regulated microRNA-378a Targets TGF-beta2 in TGF-beta1-Treated Hepatic Stellate Cells. Cell Physiol Biochem 2016;40:183-194. 